\title{
RESPOSTAS AUTONÔMICAS CARDÍACAS À MANOBRA DE TILT EM ADOLESCENTES OBESOS
}

\author{
Antônio Fernando Brunetro*, Bruno Tesini Roseguini, Bruno Moreira Silva, Daniel Müller Hirai, Dartagnan Pinto Guedes
}

Trabalho realizado nos departamentos de Fisioterapia e Educação Física da Universidade Estadual de Londrina, PR.

*Correspondência:

Rua Vasco da Gama, 128,

Aeroporto, Londrina, PR,

Cep 86036-010

Tel: (43) 3337-2906

brunetto@uel.br

\begin{abstract}
RESUMO
InTRoDução. A obesidade está associada às alterações do sistema nervoso autônomo. Estudos prévios em adultos têm mostrado que obesos apresentam resposta autonômica cardíaca diminuída frente a mudanças posturais. Contudo, pouco se sabe sobre o impacto do sobrepeso na adolescência quanto às respostas autonômicas ao estresse postural passivo. OBjetivo. Comparar as respostas autonômicas cardíacas à manobra de tilt entre adolescentes obesos e não-obesos mediante análise da variabilidade da freqüência cardíaca (VFC).

Métodos. Quatorze adolescentes obesos (15,5 $\pm 1,6$ anos) foram comparados com 20 adolescentes não-obesos ( $15,4 \pm 0,8$ anos). A modulação autonômica cardíaca foi estudada mediante análise da variabilidade da freqüência cardíaca de trechos de cinco minutos de registro de intervalos RR antes e depois da inclinação da mesa de tilt a $70^{\circ}$. A VFC foi estudada no domínio do tempo (DT) e no domínio da frequeência (DF). Os componentes espectrais foram estudados na baixa (LF) e na alta freqüência (HF) e pela razão LF/HF.

Resultados. Adolescentes obesos demonstraram menor HF em unidades normalizadas $(38,2 \pm 11$. I vs $53,9 \pm 15,5$, $p<0,05)$ e maior LF em unidades normalizadas $(60,7 \pm 11,3$ vs $44,6 \pm 15,7, p<0,05)$ na posição supina. Nenhuma diferença foi encontrada nos parâmetros da VFC após a manobra de tilt. Ao comparar a diferença entre as posições ortostática e supino, os adolescentes obesos apresentaram menores mudanças de LF unidades normalizadas (22,4 12,6 vs $38 \pm 16,4, p<0,05)$ e $H F$ unidades normalizadas $(-21,9 \pm 12,4$ vs $-37,3 \pm 16,3, p<0,05)$.
\end{abstract}

Conclusão. Adolescentes obesos apresentaram uma resposta autonômica cardíaca alterada frente ao estresse postural, caracterizada principalmente por uma hiporesponsividade vagal.

UnITERMos: Obesidade. Adolescência. Sistema nervoso autonômo.

\section{INTRODUÇÃO}

A obesidade na adolescência está associada à maior ocorrência de disfunções cardiovasculares e distúrbios metabólicos na idade adulta'. De acordo com o Harvard Growth Study, adolescentes obesos do sexo masculino apresentam um risco relativo de morte por doença arterial coronária na idade adulta duas vezes maior que adolescentes não-obesos. Contudo, os mecanismos fisiopatológicos responsáveis por essa associação ainda não estão claramente elucidados ${ }^{2}$. Vários relatos têm procurado demonstrar que um desequilíbrio no sistema nervoso autônomo (SNA), sobretudo no sistema nervoso autônomo simpático (SNS), representa um importante mecanismo tanto para o desenvolvimento da obesidade quanto de distúrbios associados ${ }^{3-7}$.

A variabilidade da freqüência cardíaca (VFC) vem sendo utilizada como ferramenta investigativa simples e não-invasiva direcionada à detecção e ao estudo das disfunções autonômicas cardíacas em diversas patologias, entre elas a obesidade ${ }^{8}$. Estudos em adultos obesos, que utilizaram a VFC, mostram que a atividade parassimpática ${ }^{9}$, ou tanto a atividade simpática quanto a parassimpática ${ }^{10,11}$, estão diminuídas nos obesos quando comparadas com os nãoobesos. Grassi et al.12, ao mensurar a atividade simpática para o músculo (MSNA - muscle sympathetic nerve activity) mediante a técnica de microneurografia, mostraram que os sujeitos obesos apresentam uma atividade simpática aumentada. Picirillo et al. ${ }^{13}$, estudando a resposta autonômica cardíaca diante do estresse postural passivo (tilt), concluíram que adultos obesos apresentam hiporesponsividade, principalmente do SNS, às mudanças posturais, sugerindo que a obesidade pode estar relacionada a alterações nos mecanismos autonômicos responsáveis pelos ajustes cardiovasculares ao estresse ortostático.

A manobra de tilt é reconhecidamente um estímulo relevante clínico e fisiológico para o estudo do SNA e pode ser utilizada para a detecção precoce de distúrbios autonômicos em diversas patologia ${ }^{14,15,16}$. Contudo, ao contrário da população adulta obesa ${ }^{13,17}$, pouco se conhece com relação às respostas cardiovasculares ao tilt em adolescentes obesos. Estudos que utilizaram a VFC mostram que a obesidade na adolescência está claramente associada a disfunções tanto do SNS quanto do SNP em repouso 18,19,20. Entretanto, diferentemente das coletas de informações em repouso, a manobra de tilt permite a realização de uma avaliação dinâmica dos mecanismos regulatórios do sistema cardiovascular. Dessa forma, o objetivo do presente estudo foi comparar, mediante análise da VFC, as respostas autonômicas cardíacas diante da manobra de tilt entre adolescentes obesos e não-obesos. 


\section{Métodos}

Trinta e quatro adolescentes obesos e não-obesos do sexo masculino, com idades entre 13 e 18 anos, foram aleatoriamente recrutados em escolas públicas locais. Todos os sujeitos completaram um questionário a respeito da história médica pregressa, consumo de bebidas alcóolicas, uso de tabaco e nível de prática habitual de atividade física. Nenhum dos adolescentes envolvidos na amostra apresentou história de doenças cardiovasculares e pulmonares, diabetes, síncope ou relataram estar utilizando qualquer tipo de medicamento. Os adolescentes e seus respectivos pais e/ou responsáveis foram informados quanto aos objetivos do estudo e apresentaram, por escrito, consentimento para participação. O protocolo de intervenção no estudo foi aprovado pelo Comitê de Ética em Pesquisa da Universidade Estadual de Londrina e o acompanham normas da Resolução 196/96 do Conselho Nacional de Saúde sobre pesquisa envolvendo seres humanos.

Após mensuração do peso corporal e da estatura, mediante técnica convencional, foi calculado o Índice de Massa Corpórea (IMC) dos adolescentes, dividindo-se a medida referente ao peso corporal $(\mathrm{kg})$ pela medida ao quadrado da estatura $(\mathrm{m})$. Foram considerados obesos aqueles adolescentes que apresentavam $\mid M C>27 \mathrm{~kg} / \mathrm{m}^{2}$. As características físicas dos adolescentes obesos $(n=14)$ e não-obesos $(n=20)$ são apresentadas na Tabela I.

Nos dias em que foram coletadas as informaç̧ões para o estudo, os sujeitos foram instruídos a não consumir bebidas a base de cafeína e nenhum tipo de bebida alcóolica. Os adolescentes analisados também foram orientados no sentido de não realizarem atividades físicas extenuantes a partir do dia anterior à coleta de dados.

\section{Protocolo}

Todos os procedimentos do estudo foram realizados entre 14 h00 e I6h00, após uma refeição leve, em uma sala silenciosa com temperatura ambiente entre $20^{\circ}$ e $23^{\circ} \mathrm{C}$.

Após exposição dos procedimentos e colocação do freqüencímetro cardíaco para monitoração da frequêencia cardíaca (FC) e do esfigmomanômetro para mensuração da pressão arterial (PA), os adolescentes foram posicionados em uma mesa ortostática (mesa de tilt). Inicialmente os adolescentes permaneceram por 25 minutos na posição supina, sendo que os primeiros 15 minutos foram considerados como período de estabilização da FC e da PA. Após os primeiros 25 minutos, a mesa de tilt foi inclinada manualmente para $70^{\circ} \mathrm{em}$ aproximadamente 2 a 3 segundos, e os adolescentes permaneceram nessa posição por mais 10 minutos. A pressão arterial foi mensurada por método auscultatório convencional em supino, após o período de estabilização, e na posição ortostática, imediatamente após a inclinação da mesa de tilt. Durante todo o período de registro, os adolescentes foram instruídos a respirar em uma freqüência de $0,25 \mathrm{~Hz}$ (I5 ciclos/ $\mathrm{min}$ ), acompanhando o sinal de um metrônomo, para garantir que as variações de $\mathrm{FC}$ relacionadas à respiração não se sobrepusessem às variações que ocorrem na baixa-freqüência da análise de potência espectral $(<0,15 \mathrm{~Hz})^{21}$.

\section{Análise da variabilidade da freqüência cardíaca}

$O$ registro dos intervalos RR batimento-a-batimento foi realizado durante todo o protocolo com frequêencia de amostragem de $1000 \mathrm{~Hz}$,

\begin{tabular}{|c|c|c|c|}
\hline \multicolumn{4}{|c|}{$\begin{array}{l}\text { Tabela I - Média e desvio padrão de características } \\
\text { antropométricas dos adolescentes analisados }\end{array}$} \\
\hline & $\begin{array}{l}\text { Não-obesos } \\
(n=20)\end{array}$ & $\begin{array}{l}\text { Obesos } \\
(n=14)\end{array}$ & Testet \\
\hline $\begin{array}{l}\text { Idade (anos) } \\
\text { Peso corporal (Kg) } \\
\text { Estatura }(\mathrm{cm}) \\
\text { Índice de massa } \\
\text { corpórea }\left(\mathrm{kg} / \mathrm{m}^{2}\right)\end{array}$ & $\begin{array}{c}15,4 \pm 0.8 \\
60,8 \pm 8,9 \\
172 \pm 7 \\
20,5 \pm 1,9\end{array}$ & $\begin{array}{c}15,5 \pm 1,6 \\
92,8 \pm 10,7 \\
173 \pm 7 \\
30,9 \pm 3,6\end{array}$ & $\begin{array}{c}\text { ns } \\
p<0,001 \\
\text { ns } \\
p<0,001\end{array}$ \\
\hline
\end{tabular}

utilizando-se freqüencímetro marca Polar ${ }^{\circledast}$, modelo $\$ 8 / 0$. Previamente à análise da VFC, todos os registros foram inspecionados visualmente na tentativa de garantir que nenhum artefato viesse a contaminar as informações coletadas. Os intervalos RR que diferiram \pm 20 batimentos em relação ao valor médio foram submetidos a filtragem ${ }^{22}$.

Foi selecionado um trecho estável de cinco minutos de registro dos intervalos RR na posição supina e outro na posição ortostática, sendo que os primeiros dois minutos após a manobra de tilt não foram considerados para análise. A análise da VFC no domínio do tempo (DT) e no domínio da freqüência (DF) foi realizada por intermédio de métodos descritos na literatura ${ }^{23}$. A análise no DT foi realizada mediante cálculo do desvio padrão dos intervalos RR (SDRR) e pela raiz quadrada do valor médio da soma dos quadrados das diferenças entre intervalos RR sucessivos (rMSSD). Para o DF os intervalos RR foram inicialmente interpolados a $4 \mathrm{~Hz}$ com uma função cúbica "B-spline", seguido da eliminação de tendências lineares ${ }^{22}$ e, por fim, foi aplicada a Transformada Rápida de Fourier (FFT) (janela de Hanning). Baseando-se na densidade espectral da FFT foram calculadas as potências das bandas de muito-baixa freqüência $(V L F)(\leq 0,04 \mathrm{~Hz})$, baixa freqüência (LF) $(0,04-0,15 \mathrm{~Hz})$, alta freqüência $(\mathrm{HF})(0,15-0,4 \mathrm{~Hz})$ e potência total $(0-0,04 \mathrm{~Hz})$. A análise espectral foi realizada mediante software específico de técnicas de análise de séries temporais (TSAS) desenvolvido por Yamamoto e Hughson ${ }^{24}$. As potências de LF, HF e da razão LF/ HF foram consideradas como marcadores simpático e parassimpático, parassimpático e do balanço simpato-vagal, respectivamente ${ }^{23}$. A potência de LF e a de HF foram estudadas em unidades normalizadas (nu), mediante a fórmula:

$$
P /(T P-V L F) \times 100
$$

onde $\mathrm{P}$ refere-se à potência absoluta de LF ou HF (em ms²), TP à potência espectral total (em $\mathrm{ms}^{2}$ ) e VLF à potência espectral da banda de muito-baixa freqüência (em ms $\left.{ }^{2}\right)$. A amplitude da resposta $\left(\triangle_{\text {difi }}\right)$ induzida pela manobra de tilt foi também calculada mediante a subtração do valor de tilt do valor em supino para todas as variáveis do DT e do DF.

\section{Análise estatística}

Todos os procedimentos estatísticos foram realizados utilizando-se do pacote estatístico Statistical Package for the Social Science, versão I0.0 para Windows (SPSS Inc, Chicago, IL). Para comparação das características antropométricas dos adolescentes obesos e não-obesos foi utilizado o teste $t$ de Student para amostras independentes. Para verificar eventuais diferenças entre os grupos em relação aos 
Tabela 2 - Valores médios e desvios padrão associados à variabilidade da freqüência cardíaca no domínio do tempo e da freqüência e à pressão arterial na posição supina e na posição ortostática (tilt)

\begin{tabular}{|c|c|c|c|c|}
\hline & & Não-obeso & Obeso & Teste F \\
\hline \multirow{3}{*}{$\operatorname{RR}(\mathrm{ms})$} & Supino & $858,9 \pm \mid 23,1$ & $888,1 \pm \mid 30,4$ & $\overline{n s}$ \\
\hline & Ortostatismo & $724,3 \pm 88,4$ & $760,6 \pm 108,8$ & ns \\
\hline & $\triangle_{\mathrm{d}}$ & $-134,5 \pm 67,5$ & $-127,5 \pm 54$ & ns \\
\hline \multirow[t]{3}{*}{$\operatorname{PAS}(\mathrm{mmHg})$} & Supino & $110,5 \pm 8,2$ & $118,5 \pm 12,3$ & 0,02 \\
\hline & Ortostatismo & $106,7 \pm 5,6$ & $\mid 13,2 \pm 1,4$ & ns \\
\hline & $\Delta_{\mathrm{dff}}$ & $-3,7 \pm 7,2$ & $-5,3 \pm 10$ & ns \\
\hline \multirow[t]{3}{*}{$\operatorname{PAD}(\mathrm{mmHg})$} & Supino & $64,5 \pm 7,76$ & $65,3 \pm 12$ & ns \\
\hline & Ortostatismo & $72,2 \pm 10$ & $69,6 \pm 14$ & ns \\
\hline & $\triangle_{d x}$ & $7,7 \pm 6,9$ & $4,2 \pm 4,7$ & ns \\
\hline \multirow[t]{3}{*}{ SDRR(ms) } & $\begin{array}{l}\text { Supino } \\
\text { of }\end{array}$ & $53,6 \pm 12,7$ & $64,1 \pm 31$ & ns \\
\hline & Ortostatismo & $49,5 \pm 18$ & $60,7 \pm 19,6$ & ns \\
\hline & $\Delta_{\mathrm{dft}}$ & $-4, \mid \pm 21,3$ & $-3,3 \pm 30,8$ & ns \\
\hline \multirow[t]{3}{*}{ RMSSD (ms) } & $\begin{array}{l}\text { Supino } \\
\text { of }\end{array}$ & $72,8 \pm 27,8$ & $66,7 \pm 39$ & ns \\
\hline & Ortostatismo & $20,7 \pm 11$ & $32,5 \pm 20$ & ns \\
\hline & $\Delta_{\text {d }}$ & $-52,1 \pm 25,5$ & $-34,1 \pm 39,9$ & ns \\
\hline \multirow[t]{3}{*}{$\mathrm{TP}\left(\mathrm{ms}^{2}\right)$} & Supino & $1449,7 \pm 689,9$ & $2622,3 \pm 3379$ & 0,04 \\
\hline & Ortostatismo & $\mid 174,1 \pm 876,2$ & $1977,5 \pm$ | | 63,1 & ns \\
\hline & $\Delta_{\mathrm{d}}$ & $-2275,6 \pm 1077,4$ & $-644,8 \pm 3002,9$ & ns \\
\hline \multirow[t]{3}{*}{$\operatorname{LF}(n u)$} & $\begin{array}{l}\text { Supino }\end{array}$ & $44,6 \pm 15,7$ & $60,7 \pm 11,3$ & 0,02 \\
\hline & Ortostatismo & $82,7 \pm 11,9$ & $83,2 \pm 7,1$ & ns \\
\hline & $\Delta_{\mathrm{dff}}$ & $38 \pm 16,4$ & $22,4 \pm 12,6$ & 0,006 \\
\hline \multirow[t]{3}{*}{$H F(n u)$} & $\begin{array}{l}\text { Supino } \\
\text { of }\end{array}$ & $53,9 \pm 15,5$ & $38,2 \pm 11,1$ & 0,02 \\
\hline & Ortostatismo & $16,5 \pm 11,9$ & $16,3 \pm 6,9$ & ns \\
\hline & $\triangle_{\mathrm{dff}}$ & $-37,3 \pm 16,3$ & $-21,9 \pm 12,4$ & 0,006 \\
\hline \multirow[t]{3}{*}{ LF/HF } & Supino & $1,04 \pm 0,84$ & $2,46 \pm 3,62$ & ns \\
\hline & Ortostatismo & $7,2 \pm 4,1$ & $6,3 \pm 3,5$ & ns \\
\hline & $\Delta_{d f}$ & $6,2 \pm 4.2$ & $3,8 \pm 4,6$ & ns \\
\hline
\end{tabular}

RR (R-R média dos intervalos RR), PAS (pressão arterial sistólica), PAD (pressão arterial diastólica), SDRR (desvio padrão dos intervalos R-R), MSSD (raiz quadrada da média da diferença entre intervalos RR sucessivos), TP (potência espectral total), LF (potência da banda de baixa freqüência em unidades normalizadas units), HF (potência da banda de alta-freqüência em unidades normalizadas), LF/HF (razão entre a banda de baixa e alta frequiência)

parâmetros da VFC, recorreu-se à análise de variância para medidas repetidas. Quando os valores de F associados à ANOVA foram estatisticamente significativos, recorreu-se ao teste de comparações múltiplas post hoc de Newman-Keuls.

\section{Resultados}

As características antropométricas dos adolescentes analisados no estudo são apresentadas na Tabela I. Como esperado, aqueles adolescentes considerados como obesos apresentaram valores médios associados ao peso corporal e ao IMC mais elevados $(p<0.00 \mathrm{I})$ que os adolescentes considerados não-obesos.

Os valores médios quanto à VFC e às medidas de pressão arterial na posição supina e em tilt são apresentados na Tabela 2. A manobra de tilt induziu a uma diminuição nas medidas observadas de pressão arterial sistólica $(p=0,004)$ e a um aumento nas medidas de pressão arterial diastólica $(p<0,00 \mathrm{l})$ entre os adolescentes obesos e nãoobesos. Observou-se também um aumento de LFnu $(p<0,00 \mathrm{I})$ e na razão $\mathrm{LH} / \mathrm{HF}(p<0,00 \mathrm{I})$, e uma diminuição de $R R(p<0,00 \mathrm{I})$, HFnu $(p<0,00$ I) e RMSSD $(p<0,00 I)$ em resposta ao tilt em ambos os grupos.
Em posição supina, os adolescentes obesos apresentaram valores médios mais elevados quanto às medidas de pressão arterial sistólica $(p=0,02), \operatorname{TP}(p<0,04)$ e LFnu $(p<0,02)$, e menores valores médios com relação ao HFnu $(p<0,02)$. Na posição ortostática, não foram observadas diferenças significativas em nenhuma variável do DT, da DF e nas medidas de pressão arterial. Ao analisar o $\triangle_{\text {dif }}$ de resposta ao tilt, verifica-se que os adolescentes obesos apresentaram menores valores para o LFnu $(p<0,00 I)$ e o HFnu $(p<0,00 I)$ quando comparados com os adolescentes não-obesos. Os valores médios de LFnu e HFnu nas posições supina e ortostática dos adolescentes analisados são ilustrados na Figura I. Tanto os adolescentes obesos como os não-obesos apresentaram diferenças significativas em linguagem estatística em ambos os indicadores de VFC. No caso do LFnu, os valores médios mais elevados foram observados na posição ostostática, enquanto no caso do HFnu, os valores médios mais elevados foram constatados na posição supina.

\section{Dıscussão}

O principal achado do presente estudo refere-se ao fato dos adolescentes obesos analisados terem apresentado uma 


\section{Figura I - Comparação dos valores médios associados ao LF (nu) e ao HF (nu) entre os adolescentes não-obesos e obesos em posições supina (branco) e ortostática (cinza)}
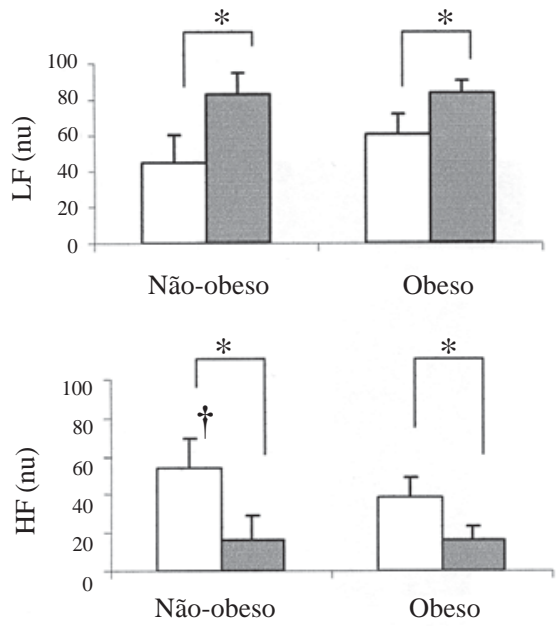

hiporesponsividade autonômica cardíaca ao estresse ortostático quando comparados com os adolescentes não-obesos. Esse resultado reforça a hipótese de que um eventual distúrbio precoce nos sistemas dinâmicos de regulação cardiovascular pode estar associado ao desenvolvimento de doenças cardiovasculares na idade adulta ${ }^{2}$.

Estudos relacionados à função autonômica cardíaca em adolescentes obesos têm mostrado resultados controversos. Rabbia et al. ${ }^{19} \mathrm{e}$ Riva et al. ${ }^{20}$ sugerem que os adolescentes obesos podem apresentar uma disfunção simpato-vagal caracterizada por redução da atividade parassimpática e aumento da atividade simpática. Yakinci et al. ${ }^{25}$ propõem que possa existir apenas uma hipoatividade vagal, enquanto Nagai et al. ${ }^{18}$ sugerem que tanto a atividade vagal quanto a simpática tendem a estarem reduzidas no jovem obeso. A discrepância entre estes resultados pode estar relacionada às diferentes metodologias empregadas, principalmente ao comparar coletas de dados realizadas em intervalos de tempo de curta ou de longa duração. A análise espectral de coletas de dados realizadas em espaço de tempo de longa duração está associada a problemas de estacionaridade dos dados, e pode, desse modo, obscurecer informações relacionadas à modulação autonômica cardíaca nessas condições ${ }^{23}$.

No presente estudo, em posição supina, foram encontrados valores médios mais baixos quanto ao HFnu e mais elevados com relação ao LFnu entre os adolescentes obesos, o que corresponde a uma menor atividade vagal e possivelmente a uma maior atividade simpática, respectivamente. Contudo, embora alguns autores proponham que a potência de LF em unidades normalizadas possa ser um marcador da atividade simpática cardíaca ${ }^{24}$, tem sido amplamente sugerido que a potência espectral dessa banda reflete tanto a atividade vagal quanto a atividade simpática ${ }^{23,27}$.

Embora não tenha sido encontrada nenhuma diferença na posição ortostática para as variáveis da VFC, a amplitude das respostas ao tilt associada ao HFnu e ao LFnu foi menor entre os adolescentes obesos, demonstrando possivelmente uma hiporesponsividade simpática e vagal ao estresse ortostático. Contudo, considerando que tanto o valor de LFnu em supino quanto a amplitude da resposta de LFnu ao tilt não foram acompanhados simultaneamente por uma diferença nos valores de LH/HF, que reflete o balanço simpato-vagal, pode-se tão-somente especular quanto à eventual presença de uma disfunção do SNS simpático nesses jovens.

A resposta alterada ao tilt, principalmente vagal, em adolescentes obesos pode oferecer importante contribuição para o entendimento fisiopatológico da obesidade, bem como sugerir indicadores de prognósticos. A mudança postural passiva ocasiona uma diminuição do retorno venoso e conseqüente aumento da freqüência cardíaca induzido pela retirada da atividade vagal e ativação simpática mediada pelos baroreceptores arteriais e cardiopulmonares ${ }^{16}$. Assim como em adultos obesos, que apresentam respostas alteradas ao tilt ${ }^{13,17}$, os resultados do presente estudo podem ser explicados baseando-se em possível disfunção no controle baroreflexo da freqüência cardíaca associada à obesidade ${ }^{12}$. Como o baroreflexo modula a freqüência cardíaca principalmente pela atividade vagal ${ }^{28}$, é possível que a menor retirada vagal possa sugerir uma disfunção precoce nesse sistema.

Provável distúrbio no controle baroreflexo, associado à menor atividade vagal em repouso nos adolescentes obesos, pode oferecer subsídios quanto à relação entre a obesidade instalada na adolescência e a ocorrência de disfunções cardiovasculares na idade adulta. Vários relatos mostram que a diminuição da modulação vagal para o coração apresenta estreita relação com distúrbios cardíacos como arritmias ventriculares e morte súbita ${ }^{29,30}$. Portanto, considerando a acentuada prevalência desta patologia, faz-se necessário o uso de estratégias reabilitavas e preventivas que possivelmente possam tentar reverter essa tendência de forma efetiva.

Em conclusão, os resultados aqui apresentados sugerem que adolescentes obesos podem apresentar resposta autonômica cardíaca alterada ao estresse postural, caracterizada principalmente por uma hiporesponsividade vagal.

\section{Conflito de interesse: não há.}

\section{SUMMARY}

\section{Cardiac autonomic responses to head-up tilt in Obese ADOLESCENTS}

INTRODUCTION. Obesity is associated with alterations of the autonomic nervous system. Previous studies in adults have shown that obese individuals present a decreased cardiac autonomic response to postural challenges. However, little is known about the impact of overweight on autonomic responses to passive postural stress in adolescents.

OBJECTIVE. to compare cardiac autonomic responses to the head-up tilt maneuver between obese and non-obese adolescents by analyzing heart rate variability.

METHODS. Fourteen obese adolescents (15.5 \pm 1.6 years) were compared with twenty non-obese subjects ( $15.4 \pm 0.8$ years). Cardiac autonomic modulation was studied by heart rate variability (HRV) analysis of 5 minute $R R$ interval recordings before and after a $70^{\circ}$ head-up tilt 
BRUNETTO AF ET AL.

maneuver. HRV was analyzed according to the time domain (TD) and frequency-domain (FD) methods. The power spectral components were studied at low (LF) and high (HF) frequencies and as the LF/HF ratio.

REsULTS. Obese adolescents demonstrated significantly lower HF normalized units $(38.2 \pm 11$. I vs $53.9 \pm 15.5, p<0.05)$ and higher $L F$ normalized units $(60.7 \pm 11.3$ vs $44.6 \pm 15.7, p<0.05)$ in the supine position. No difference was found in any HRV parameters after head-up tilt. When comparing differences between the orthostatic and supine positions, obese adolescents showed lesser changes of $L$ F normalized units $(22.4 \pm 12.6$ vs $38 \pm 16.4, p<0.05)$ and HF normalized units $(-21.9 \pm 12.4$ vs $-37.3 \pm 16.3, p<0.05)$.

CONCLUSIONs. Obese adolescents have an abnormal cardiac autonomic response to the head-up tilt maneuver, characterized principally by a decreased parasympathetic response. [Rev Assoc Med Bras 2005; 5I(5): 256-60]

KEY words: Obesity. Adolescence. Autonomic Nervous System.

\section{RefERÊNCIAS}

I. Dietz, WH. Childhood weight affects adult morbidity and mortality. J Nutr 1998; | 2:4| IS-4S.

2. Must A, Jacques PF, Dallal GE, Bajema CJ, Dietz W. Long-term morbidity and mortality of overweight adolescents. A Follow-up of the Harvard Grownth Study of 1922 to 1935. N Engl J Med 1992;327: I350-5.

3. Landsberg L. Obesity and the insulin resistance syndrome. Hypertens Res 1996; 19:S5I-S5.

4. Scherrer U, Randin D, Tappy L, Vollenweider P, Jéquier E, Nicod P. Body fat and sympathetic nerve activity in healthy subjects. Circulation 1994;89:2634-40.

5. Tuck ML. Obesity, the symphathetic nervous system, and essential hypertension. Hypertension 1992; I9(Suppl I):67-77.

6. Kuniyoshi FH, Trombetta IC, Batalha LT, Rondon MU, Laterza MC, Gowdak MM, et al. Abnormal neurovascular control during sympathoexcitation in obesity. Obes Res 2003; | | | | 4 | |-9.

7. Ribeiro MM, Trombetta IC, Batalha LT, Rondon MU, Forjaz CL, Barretto $A C$, et al. Muscle sympathetic nerve activity and hemodynamic alterations in middle-aged obese women. Braz J Med Biol Res $2001 ; 34: 475-8$.

8. Montano N. Heart rate variability as a clinical tool. Ital Heart J 2002;3:43945.

9. Zahorska-Markiewicz B, Kuagowsa E, Kucio C, Klin M. Heart rate variability in obesity. Int J Obes Relat Metab Disord 1993; 17:21-3.

10. Peterson HU, Rothschid M, Weinberg CR, Fell RD, McLeish KR, Pfeifer $M$. Body fat and the activity of the autonomic nervous system. N Eng J Med 1988;318:1077-83

I I. Laederach-Hofmann K, Mussgay L, Rúddel H. Autonomic cardiovascular regulation in obesity. J Endocrinol 2000; 164:59-66.

12. Grassi G, Seravalle G, Cattaneo BM, Bolla GB, Lanfranchi A, Colombo M, et al. Sympathetic activation in obese normotensive subjects (part I). Hypertension 1995;25:560-3.

13. Piccirillo G, Vetta F, Figognari FL, Ronzoni S, Lama J, Cacciafesta M, et al. Power spectral analysis of heart rate variability in obese subjects: evidence of decreased cardiac sympathetic responsiveness. Int J Obes Relat Metab Disord 1996; 20:825-9.

14. Lipsitz L, Mietus J, Moody G, Goldberger A. Spectral characteristics of heart rate variability before and during postural tilt. Relation to age and risk of syncope. Circulation 1990;81: 1803-10.

15. Montano N, Ruscone T, Porta A, Lombardi F, Pagani M, Malliani A. Power spectrum analysis of heart rate variability to assess the changes in sympathovagal balance during graded orthostatic tilt. Circulation |994;90: | 826-3|.

16. Mukai S, Hayano J. Heart rate and blood pressure variabilities during graded head-up tilt. J Appl Physiol 1995;78:212-6.

17. Zahorska-Markiewicz B, Mizia-Stec K, Jastrzebska-Maj E, Mandecki T, Bilewicz-Wyrozumska T, Mucha Z, et al. Tilt tabe testing in obesity. Int J Cardiol 2003;88:43-8

18. Nagai N, Matsumoto T, Kita H, Moritani T. Autonomic nervous system activity and the state and development of obesity in japanese school children. Obes Res 2003; 1 1:25-32.

19. Rabbia F, Silke B, Conterno A, Grosso T, De Vito B, Rabbone I, et al. Assessment of cardiac autonomic modulation during adolescent obesity. Obes Res 2003; I 1:541-8.

20. Riva P, Martini G, Rabbia F, Milan A, Paglieri C, Chiandussi L, et al. Obesity and autonomic function in adolescence. Clin Exp Hypertens 200 I;23( I2):57-67.

21. Ori Z, Monir G, Weiss J, Sayhouni X and Singer DH. Heart rate variability: frequency domain analysis. Cardiol Clin 1992; 10:499-533.

22. Yamamoto Y, Hughson RL, Peterson J. Autonomic control of heart rate during exercise studied by heart rate variability spectral analysis. J Appl Physiol 1991;71: I 136-42.

23. Task Force of the European Society of Cardiology and the North American Society of Pacing and Electrophysiology. Heart rate variability: standards of measurements, physiological interpretation and clinical use. Circulation 1996;93:1043-65.

24. Yamamoto $Y$, Hughson RL. Extracting fractal components from time series. Physica D 1993; 68:250-64.

25. Yakinci C, Mungen B, Karabiber H, Tayfun M, Evereklioglu C. Autonomic nervous system functions in obese children. Brain Dev 2000;22: I 5 I-3.

26. Montano N, Porta A, Malliani A. Evidence for central organization of cardiovascular rhythms. Ann N Y Acad Sci USA 2001;940:299-306.

27. Houle MS, Billman GE. Low-frequency component of heart rate variability spectrum: a poor marker of sympathetic activity. Am J Physiol 1999;276:H2 I5-H23.

28. Mancia G, Mark AL. Arterial baroreflexes in humans. In: Shepherd JT, Abboud FM, editors Handbook of physiology: Bethesda: American Physiological Society; 1983. p.755-93. (Section 2: the cardiovascular system, III).

29. Stys A, Stys T. Current clinical applications of heart rate variability. Clin Cardiol 1998;21:719-24.

30. Routledge HC, Chowdhary S, Townend JN. Heart rate variability: a therapeutic target? J Clin Pharmacol Ther 2002; 27:85-92. 